DRAFT VERSION OCTOBER 23, 2018

Preprint typeset using LTEX style emulateapj v. 11/10/09

\title{
TESTING MODIFIED NEWTONIAN DYNAMICS WITH ROTATION CURVES OF DWARF AND LOW SURFACE BRIGHTNESS GALAXIES
}

\author{
R. A. SWATERS ${ }^{1,2}$ \\ Department of Astronomy, University of Maryland, College Park, MD 20742-2421 \\ R. H. SANDERS \\ Kapteyn Institute, P.O. Box 800, 9700 AV Groningen, the Netherlands \\ S. S. MCGAUGH \\ Department of Astronomy, University of Maryland, College Park, MD 20742-2421 \\ Draft version October 23, 2018
}

\begin{abstract}
Dwarf and low surface brightness galaxies are ideal objects to test modified Newtonian dynamics (MOND), because in most of these galaxies the accelerations fall below the threshold below where MOND supposedly applies. We have selected from the literature a sample of 27 dwarf and low surface brightness galaxies. MOND is successful in explaining the general shape of the observed rotation curves for roughly three quarters of the galaxies in the sample presented here. However, for the remaining quarter, MOND does not adequately explain the observed rotation curves. Considering the uncertainties in distances and inclinations for the galaxies in our sample, a small fraction of poor MOND predictions is expected and is not necessarily a problem for MOND. We have also made fits taking the MOND acceleration constant, $a_{0}$, as a free parameter in order to identify any systematic trends. We find that there appears to be a correlation between central surface brightness and the best-fit value of $a_{0}$, in the sense that lower surface brightness galaxies tend to have lower $a_{0}$. However, this correlation depends strongly on a small number of galaxies whose rotation curves might be uncertain due to either bars or warps. Without these galaxies, there is less evidence of a trend, but the average value we find for $a_{0} \approx 0.7 \times 10^{-8} \mathrm{~cm} \mathrm{~s}^{-2}$ is somewhat lower than derived from previous studies. Such lower fitted values of $a_{0}$ could occur if external gravitational fields are important.

Subject headings: galaxies: dwarfs - galaxies: kinematics and dynamics
\end{abstract}

\section{INTRODUCTION}

Modified Newtonian dynamics (MOND) was proposed by Milgrom (1983a,b) as an alternative to dark matter. MOND posits that the effective gravitational force deviates from the Newtonian force: at accelerations below a critical value, $a_{0}$, the gravitational force is proportional to the square root of the Newtonian force; at higher accelerations, the force is Newtonian. In principle, MOND predicts the shape and amplitude of the observed rotation curves from the observed mass distribution, i.e., gas and stars, with only the mass-to-light ratio $(\mathrm{M} / \mathrm{L})$ of the stellar disk as adjustable parameter.

The rotation curves of a large number of spiral galaxies have been considered in the context of MOND. For example, Begeman et al. (1991), Sanders (1996), Sanders \& Verheijen (1998), and Sanders \& Noordermeer (2007) demonstrated that for the spiral galaxies the observed rotation curves were, in most cases, predicted in detail, using the MOND prescription, from the observed light and gas distributions. Moreover, the M/Ls derived from these fits are generally astrophysically plausible and consistent with stellar population synthesis models. In a handful of these galaxies the rotation curves predicted by MOND are noticeably different from the observed rotation curves. These were not considered falsifi-

${ }^{1}$ Department of Physics and Astronomy, Johns Hopkins University, 3400 N. Charles Str., Baltimore, MD 21218

2 Space Telescope Science Institute, 3700 San Martin Dr., Baltimore, MD 21218
}

cations of MOND because for most of these galaxies the authors identified an obvious problem with the observed rotation curve (e.g., uncertain inclination or distance) or with the use of the rotation curve as a tracer of the gravitational force (e.g., disturbed velocity fields).

Although MOND appears to explain the magnitude of the discrepancy in spiral galaxies, it has has long been known that the theory predicts more matter than is observed in clusters of galaxies (e.g., Sanders 1999). In this sense the famous "bullet cluster", which has been presented as direct emprical evidence for the existence of dark matter (e.g., Clowe et al. 2006), does not pose a new problem for MOND. Additional undetected matter in some dissipationless form does seem to be required in clusters in the context of MOND. In any case, additional required matter is not formally a falsification of MOND and in no sense detracts from the success of this algorithm on the scale of galaxies.

MOND is more strongly tested by spiral galaxies with welldetermined distances because the $a_{0}$ depends inversely upon distance. Bottema et al. (2002) considered a sample of 4 spiral galaxies with well-measured rotation curves and Cepheidbased distances determined as part of the HST program on the extragalactic distance scale (Sakai et al. 2000). Two galaxies are in good agreement with MOND predictions, but for NGC 2841 and NGC 3198 the rotation curves are not in agreement with MOND, unless the distance to NGC 2841 is at least 20\% larger, and the distance to NGC 3198 is at least $10 \%$ smaller than their Cepheid distances indicate. Bottema 
TABLE 1

GALAXY PROPERTIES

\begin{tabular}{|c|c|c|c|c|c|c|c|c|c|}
\hline $\begin{array}{c}\text { Name } \\
\text { (1) }\end{array}$ & $\begin{array}{c}\text { Source } \\
\text { (2) }\end{array}$ & $\begin{array}{c}\mathrm{D}_{a} \\
\mathrm{Mpc} \\
(3)\end{array}$ & $\begin{array}{c}\mu_{0}^{R} \\
\mathrm{mag/ \prime ^{-2 }} \\
(4)\end{array}$ & $\begin{array}{c}h \\
\mathrm{kpc} \\
(5)\end{array}$ & $\begin{array}{c}M_{R} \\
\operatorname{mag} \\
(6)\end{array}$ & $\begin{array}{c}M_{\mathrm{HI}} \\
10^{8} M_{\odot} \\
(7)\end{array}$ & $\begin{array}{c}i \\
\circ \\
(8)\end{array}$ & $\begin{array}{c}v_{\text {rot }} \\
\mathrm{km} \mathrm{s}^{-1} \\
(9)\end{array}$ & $\begin{array}{c}a_{\operatorname{lmp}} / a_{0} \\
\text { (10) }\end{array}$ \\
\hline UGC 731 & SMBB & 8.0 & 23.0 & 1.65 & -16.6 & 7.37 & 57 & 74 & 0.25 \\
\hline UGC 3371 & SVBA & 12.8 & 23.3 & 3.09 & -17.7 & 12.2 & 49 & 86 & 0.23 \\
\hline UGC 4173 & $\mathrm{dBB}$ & 18.0 & 24.3 & 4.77 & -18.0 & 24.3 & 40 & 57 & 0.08 \\
\hline UGC 4325 & SMBB & 10.8 & 21.6 & 1.74 & -18.2 & 8.61 & 41 & 93 & 0.44 \\
\hline UGC 4499 & SMBB & 13.9 & 21.5 & 1.59 & -17.9 & 13.6 & 50 & 74 & 0.20 \\
\hline UGC 5005 & $\mathrm{dBB}$ & 56 & 22.9 & 4.71 & -18.8 & 33.2 & 41 & 99 & 0.11 \\
\hline UGC 5414 & SSAH & 10.7 & 21.8 & 1.59 & -17.7 & 7.42 & 55 & 61 & 0.26 \\
\hline UGC 5721 & SMBB & 6.6 & 20.2 & 0.45 & -16.6 & 6.63 & 61 & 79 & 0.28 \\
\hline UGC 5750 & dBMR & 60 & 22.6 & 5.99 & -19.6 & 11.4 & 64 & 79 & 0.09 \\
\hline UGC 6446 & SSAH & 12.8 & 21.4 & 2.00 & -18.5 & 15.4 & 52 & 80 & 0.20 \\
\hline UGC 7232 & SSAH & 3.5 & 20.2 & 0.33 & -15.3 & 0.71 & 59 & 44 & 0.62 \\
\hline UGC 7323 & SSAH & 8.7 & 21.2 & 2.35 & -19.0 & 8.48 & 47 & 86 & 0.38 \\
\hline UGC 7399 & SSAH & 8.4 & 20.7 & 0.79 & -17.1 & 7.40 & 55 & 109 & 0.35 \\
\hline UGC 7524 & SSAH & $4.6^{*}$ & 22.2 & 3.37 & -18.7 & 16.7 & 46 & 84 & 0.22 \\
\hline UGC 7559 & SSAH & $4.9^{*}$ & 23.8 & 1.02 & -14.6 & 1.71 & 61 & 33 & 0.11 \\
\hline UGC 7577 & SSAH & $2.5^{* *}$ & 22.5 & 0.60 & -14.9 & 0.42 & 63 & 18 & 0.06 \\
\hline UGC 7603 & $\mathrm{dBB}$ & 7.3 & 20.8 & 0.96 & -17.0 & 6.13 & 78 & 64 & 0.21 \\
\hline UGC 8490 & SMBB & $4.6^{*}$ & 20.5 & 0.62 & -17.1 & 7.01 & 50 & 80 & 0.21 \\
\hline UGC 9211 & $\mathrm{dBB}$ & 12.6 & 22.6 & 1.32 & -16.2 & 10.5 & 44 & 66 & 0.17 \\
\hline UGC 11707 & SMBB & 17.0 & 23.1 & 4.61 & -18.8 & 42.7 & 68 & 100 & 0.20 \\
\hline UGC 11861 & SMBB & 27 & 21.4 & 6.48 & -20.9 & 81.7 & 50 & 153 & 0.43 \\
\hline UGC 12060 & SSAH & 16.8 & 21.6 & 1.89 & -18.1 & 20.7 & 40 & 75 & 0.16 \\
\hline UGC 12632 & SSAH & 6.9 & 23.5 & 2.57 & -17.1 & 8.66 & 46 & 76 & 0.22 \\
\hline F568-V1 & SMBB & 86 & 22.8 & 3.42 & -18.8 & 28.1 & 40 & 118 & 0.24 \\
\hline F574-1 & SMBB & 103 & 22.6 & 4.60 & -19.3 & 39.7 & 65 & 100 & 0.22 \\
\hline F583-1 & dBMR & 34 & 22.0 & 1.71 & -17.5 & 19.9 & 63 & 84 & 0.15 \\
\hline F583-4 & dBMR & 52 & 22.4 & 2.89 & -17.8 & 6.30 & 55 & 70 & 0.22 \\
\hline
\end{tabular}

Note. - (1) the name of the galaxy, (2) source of the rotation curve: de Blok et al. (2001, dBMR), de Blok \& Bosma (2002; dBB), Swaters et al. (2003a; SMBB), Swaters et al. (2003b; SVBA), Swaters et al. (2009; SSAH), (3) adopted distance, taken from Swaters \& Balcells (2002), except that distances for galaxies marked with * were taken from Karachentev et al. (2003), those marked with ** were taken from Méndez et al. (2002) (4-6) surface brightness, scale length, and absolute magnitude, from Swaters \& Balcells (2002), de Blok et al. (1995), and McGaugh \& Bothun (1994), (7) HI mass, from Swaters et al. (2003), de Blok et al. (1996), and van der Hulst et al. (1993) (8) inclinations, from Swaters et al. (2004) and de Blok et al. (1996), (9) maximum rotation velocity, (10) acceleration at the last measured point of the rotation curve.

et al. (2002) argued that NGC 3198 is probably consistent with MOND within the uncertainties of the Cepheid distance. With respect to NGC 2841, Milgrom and Sanders (2008) have demonstrated that a reasonable match to the observed rotation curve can be achieved with alternative forms of the function that described the transition from the Newtonian to the MOND regime.

Dwarf and low surface brightness (LSB) galaxies provide an especially good test for MOND, because in most of these galaxies the acceleration is below the MOND threshold of $a_{0} \approx 10^{-8} \mathrm{~cm} \mathrm{~s}^{-2}$ (determined empirically from rotation curve fits, see e.g., Sanders \& McGaugh 2002) at all radii, whereas in high surface brightness spiral galaxies the acceleration is usually above the threshold in the inner parts. Milgrom (1983b) predicted that LSB galaxies should exhibit a large discrepancy between the detectable and Newtonian dynamical mass within the optical disk, and this prediction has since been confirmed (e.g., de Blok \& McGaugh 1997; McGaugh \& de Blok 1998; Swaters et al. 2000).

Apart from this general prediction, the MOND prescription is successful in predicting the detailed rotation curves of LSB galaxies as well. De Blok \& McGaugh (1998) found that for their sample of 15 LSB galaxies all rotation curves are well fitted with MOND, although for 6 galaxies modest adjustments of the inclination were necessary in order to get an acceptable match to the rotation curve. The sample presented in Sanders \& Verheijen (1998) contains 12 LSB galaxies, and most of these the rotation curves can be well explained by MOND as well.

The results for dwarf galaxies show a fairly large spread. Milgrom \& Braun (1988) concluded that the observed rotation curve of DDO 154 is in very good agreement with the rotation curve predicted by MOND. Lake (1989) considered a sample of 6 dwarf galaxies in the context of MOND and concluded that acceptable fits require systematically lower values for $a_{0}$ than were found for spiral galaxies. In addition, Lake (1989) found an apparent correlation between the asymptotic rotation velocity and the fitted value of $a_{0}$. Milgrom (1991), however, pointed out that uncertainties in inclinations and distances can well explain the fact that MOND appears to fail for these galaxies. The rotation curves of the dwarf galaxies included in the samples of Begeman et al. (1991) and Sanders (1996) also agree well with those calculated with MOND. On the other hand, Blais-Ouellette et al. (2001) reported that MOND provides poor predictions of the rotation curves of the three dwarf galaxies in their sample, and reported that a higher value of $a_{0}$ is needed to make their rotation curves derived from Fabry-Perot observations consistent with MOND. More recently, Milgrom \& Sanders (2007) concluded that the rotation curves of the four low-mass galaxies in their sample are explain well in the context of MOND.

Given this apparent spread in conclusions on whether MOND correctly predicts the rotation curves of dwarf galaxies, we here test MOND against a sample of rotation curves of 

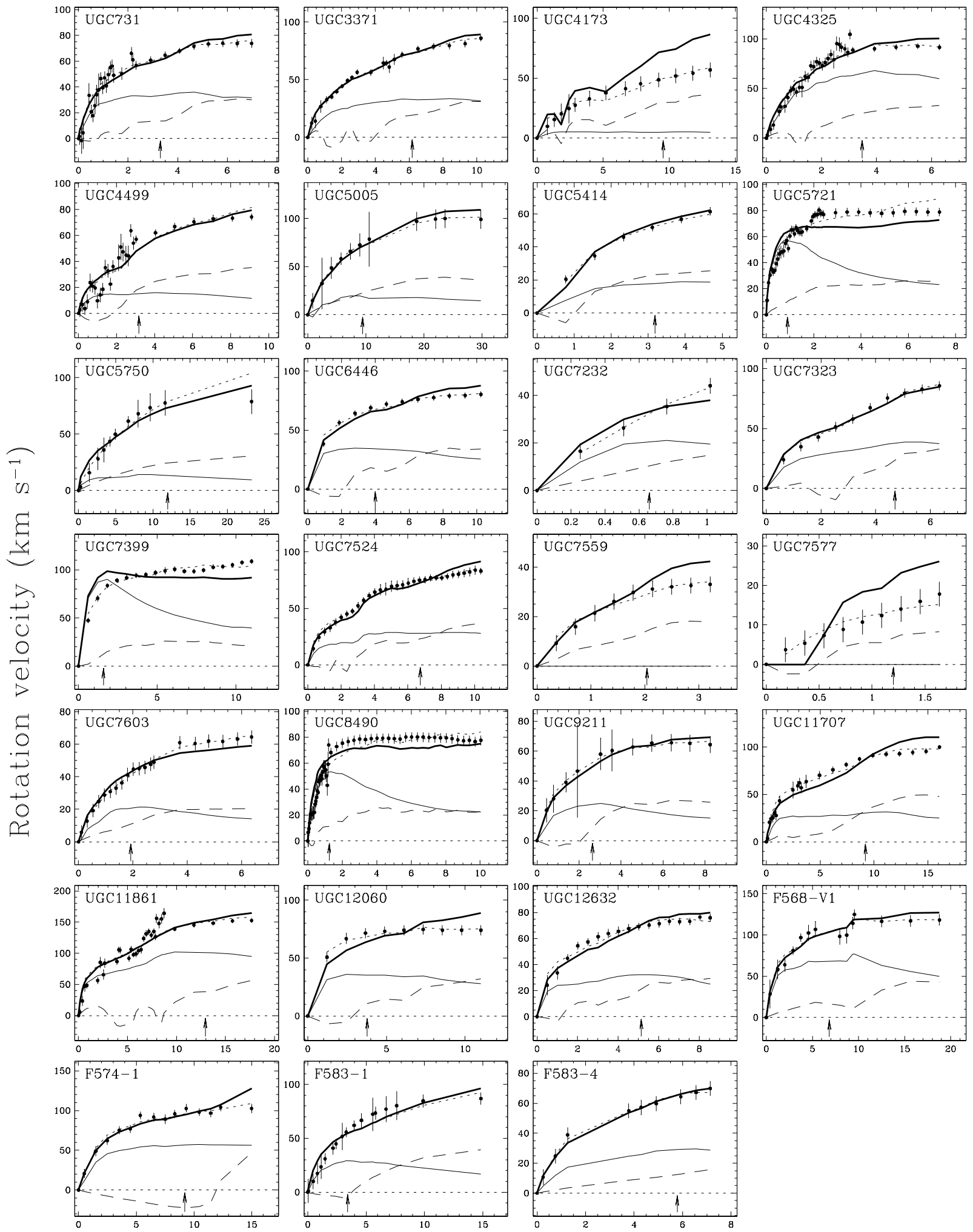

Radius (kpc)

FIG. 1.- MOND predictions. The dots represent the measured rotation velocities and their errors. The dashed line represents the contribution of the HI to the rotation curve. In cases where there is little or no $\mathrm{HI}$ at small radii in the HI disks, the HI at larger radii produces a net outward gravitational force, which is represented here by the negative velocities. The thin solid line represents the contribution of the stars. The thick solid line is the best MOND fit with only the stellar mass-to-light ratio as a free parameter. The dotted line is the best MOND fit with both the mass-to-light ratio and the distance as free parameter. 
dwarf and LSB galaxies that have recently become available in the literature. We included both dwarf and LSB galaxies because the physical properties of these galaxies are similar, although some dwarf galaxies have high surface brightness, and some LSB galaxies are large in size.

There are several difficulties inherent to such a sample. For example, because most dwarf galaxies are nearby, different methods to estimate the distance have been used, and this may introduce a dispersion in the derived parameters. Inclination is another uncertainty for a class of objects which are known to be irregular with, in some cases, large scale asymmetries. Moreover, the assumption that the rotation curve is a tracer of the radial force distribution may, itself, be questionable in cases where asymmetries are present. Bearing these considerations in mind, one might not expect, a priori, the agreement between the predicted and observed rotation curves to be as good as noted for earlier samples. A systematic failure, however, would be problematic for MOND.

The outline of this paper is as follows. In Section 2 we describe the sample used in this paper. In Section 3 we describe the fitting procedure, and in Section 4 the fitting results. Next, we comment on individual galaxies in Section 5. We describe the uncertainties in Section 6, and we discuss the results in Section 7. Finally, we present our conclusions in Section 8

\section{THE SAMPLE}

The sample we present here is compiled from data from five studies. A large fraction comes from the $\mathrm{H}$ I rotation curves of a sample of 62 dwarf galaxies (Swaters 1999; Swaters et al. 2009, hereafter SSAH). The resolution of the HI data used to derive the rotation curves is $30^{\prime \prime}$. Because of this relatively low resolution, the data have been corrected for beam smearing as described in Swaters (1999) and SSAH. From this original sample of 62 dwarf galaxies, we selected galaxies with inclination in the range $40^{\circ} \leq i \leq 80^{\circ}$, and we only included rotation curves that were classified by SSAH as high quality. Galaxies classified by SSAH as having lower quality either have a signal-to-noise ratio that is too low, or are too asymmetric to derive a reliable rotation curve. To avoid the uncertainties associated with these low quality rotation curves, we have excluded these from our sample. The resulting sample contains 19 rotation curves.

The second source is the sample of $\mathrm{H} \alpha$ rotation curves presented in Swaters et al. (2003a). They obtained high resolution rotation curves from $\mathrm{H} \alpha$ long-slit spectroscopy for a sample of 10 dwarf that are part of the SSAH sample described above, and 5 LSB galaxies from the sample presented in de Blok et al. (1996). As described in Swaters et al. (2003a), these $\mathrm{H} \alpha$ rotation curves have been combined with the $\mathrm{H} \mathrm{I}$ data presented SSAH and de Blok et al. (1996). Galaxies with $i<40^{\circ}$ or $i>80^{\circ}$ were not included in the sample here. F5683 was also excluded because of its strong bar.

The third source is the sample of LSB galaxies presented in de Blok, et al. (2001). They present long-slit $\mathrm{H} \alpha$ rotation curves for a sample of 30 LSB galaxies. From their sample we have selected those galaxies for which optical photometry and $\mathrm{H} \mathrm{I}$ imaging are available and with inclinations in the range $40^{\circ} \leq i \leq 80^{\circ}$.

The fourth source is the sample presented in de Blok \& Bosma (2002), consisting of H I-H $\alpha$ hybrid rotation curves. From this sample we have selected the galaxies that overlap with the SSAH sample. We excluded UGC 3851 because its rotation curve depends heavily on the uncertain $\mathrm{H}$ I rotation curve as derived by SSAH and which they deemed to be of low quality. UGC 7524 was also excluded because this galaxy is well resolved by the $\mathrm{H}$ I observations and the rotation curve derived from the two-dimensional data probably gives a better representation of the gravitational potential than the one derived from long-slit data. The rotation curves presented in de Blok \& Bosma (2002) have been combined with the H I rotation curves presented in SSAH as described in de Blok \& Bosma (2002).

The fifth and final source is the rotation curve of UGC 3371 presented in Swaters et al. (2003b). They obtained highresolution, two-dimensional $\mathrm{H} \alpha$ data for this galaxy.

Where the samples are overlapping, we have used them in this order of decreasing priority: Swaters et al. (2003b), Swaters et al. (2003a), de Blok \& Bosma (2002), de Blok et al. (2001), SSAH. Most rotation curves in our sample have been derived from $\mathrm{H} \alpha$ data for the central parts and $\mathrm{H}$ I data for the outer parts. However, for the 10 galaxies that came from SSAH (see column 2 in Table 1 the rotation curves are entirely based on $\mathrm{H}$ I data. We have not corrected the rotation curves in these samples for pressure support, because these corrections tend to be small in comparison to the rotation velocities, and probably uncertain (see also SSAH).

The combined sample does not constitute a complete sample of dwarf and LSB galaxies. Nonetheless, this sample contains galaxies with a wide range in luminosity and surface brightness for which high-quality rotation curve are available. This sample is therefore well suited to test MOND in late-type dwarf and LSB galaxies.

Because many of the galaxies in our sample are at small distances, their systemic velocities are a poor indicator of their distance. Where possible, we use other distance indicators, based on the compilation of distances found in the literature presented in Swaters \& Balcells (2002), supplemented with other recent distance estimates based on the tip of the red giant branch (Méndez et al. 2002; Karachentsev et al. 2003). In order of decreasing priority, the distances presented in Table 1 are based on Cepheids, the tip of the red giant branch, brightest stars, group membership, and the recession velocities corrected for Virgocentric flow as described in Swaters \& Balcells (2002). For a more complete discussion on the distances to these dwarf galaxies, see Swaters \& Balcells (2002). The galaxies in our sample that are not in the SSAH sample are all at large distances, and for these the distances have been calculated based on a Hubble constant of $70 \mathrm{~km} \mathrm{~s}^{-1} \mathrm{Mpc}^{-1}$, following Sanders \& McGaugh (2002).

\section{FITTING PROCEDURE}

To calculate the MOND rotation curves, we have used the same method as used in Sanders (1996) and Sanders \& Verheijen (1998). To calculate the contribution of the stellar component to the disk, we have assumed that the stellar mass resides in an infinitely thin disk, that the $R$-band light accurately traces the mass distribution of the stellar component, and that the stellar mass-to-light ratio is constant with radius. The gas mass distribution is also assumed to be in a thin disk. We assumed that the $\mathrm{H} \mathrm{I}$ is optically thin, and we corrected for the mass fraction of helium by scaling the $\mathrm{H}$ I mass by a factor of 1.32. The optical radial profiles were taken from Swaters \& Balcells (2002), de Blok et al. (1995) and McGaugh \& Bothun (1994). The H I radial profiles were taken from Swaters et al. (2002), de Blok et al. (1996), and van der Hulst et al. (1993). For the galaxies in which the H I distribution is poorly resolved, we calculated the radial profiles following the algorithm described by Warmels (1988) that uses a 
iterative deconvolution scheme (Lucy 1974) to correct for the effects of beam smearing (see Swaters et al. 2002 for more details). For both the stellar and the gaseous disk, we assumed that the mass distribution is well represented by the radial average.

To calculate the MOND rotation curves of the visible components, ideally the MOND field equation of Bekenstein \& Milgrom (1984) should be used. However, this is computationally expensive, and Milgrom (1986) has shown that the differences between the results derived for the field equation and those from the original MOND prescription are usually much smaller than, and practically never larger than $5 \%$. Therefore, we apply the usual MOND formula:

$$
\mu\left(g_{\mathrm{M}} / a_{0}\right) \mathbf{g}_{\mathrm{M}}=\mathbf{g}_{\mathrm{N}}
$$

where $a_{0}$ is the MOND acceleration parameter, $\mathbf{g}_{M}$ is the MOND acceleration, $\mathbf{g}_{N}$ is the Newtonian acceleration, and

$$
\mu(x)=x\left(1+x^{2}\right)^{-1 / 2},
$$

which is the commonly assumed form having the required asymptotic behaviour. For $g \gg a_{0}$ gravity is Newtonian, and for $g \ll a_{0}$ gravity is of the MOND form with $g=\left(g_{N} a_{0}\right)^{1 / 2}$. A little algebra shows that the rotation curve for MOND can be expressed as:

$$
v_{\text {rot }}^{2}=\frac{v_{\text {sum }}^{2}}{\sqrt{2}} \sqrt{1+\sqrt{1+\left(2 r a_{0} / v_{\text {sum }}^{2}\right)^{2}}} .
$$

Here, $r$ is the radius and

$$
v_{\text {sum }}^{2}=\Upsilon_{*, d} v_{d}^{2}+\Upsilon_{*, b} v_{b}^{2}+v_{g}^{2}
$$

with $v_{d}, v_{b}$ and $v_{g}$ the contribution of the stellar disk, bulge and gas to the rotation curve, respectively, calculated in the Newtonian regime as described above, and $\Upsilon_{*, d}$ and $\Upsilon_{*, b}$ are the stellar mass-to-light ratios of the disk and the bulge. Eq. 3 is fit to the observed rotation curve with a least-squares algorithm. None of the galaxies in our sample have significant bulges, which eliminates $\Upsilon_{*, b}$ as a free parameter.

It should be noted that in the context of MOND the internal dynamics of a system is affected by the external acceleration field. If the external field becomes comparable to or larger than $a_{0}$, a galaxy in this external field will always be in the Newtonian regime, even if the internal accelerations are low (Milgrom 1983a,b).

\section{RESULTS}

We made MOND fits by fitting Eq. 3 to the observed rotation curves with a least squares algorithm. Because $a_{0}$ is a universal constant in MOND, we first made fits with only $\Upsilon_{*, d}$ as a free parameter. Following e.g., Sanders \& McGaugh (2002), the acceleration parameter was fixed at $a_{0}=1.0 \times 10^{-8} \mathrm{~cm} \mathrm{~s}^{-2}$ $\left(=3080 \mathrm{~km}^{2} \mathrm{~s}^{-2} \mathrm{kpc}^{-1}\right)$.

In addition to the one parameter fits, we have also made MOND fits with the distance and inclination as free parameters in order to investigate the effects of uncertainties in these parameters. In addition, we made fits with $a_{0}$ as a free parameter to measure $a_{0}$ and look for trends.

\subsection{One-parameter MOND fits}

The one-parameter MOND fits, with only $\Upsilon_{*, d}$ as a free parameter, are shown in Figure 1 In each panel of this figure, the dots represent the measured rotation velocities and their

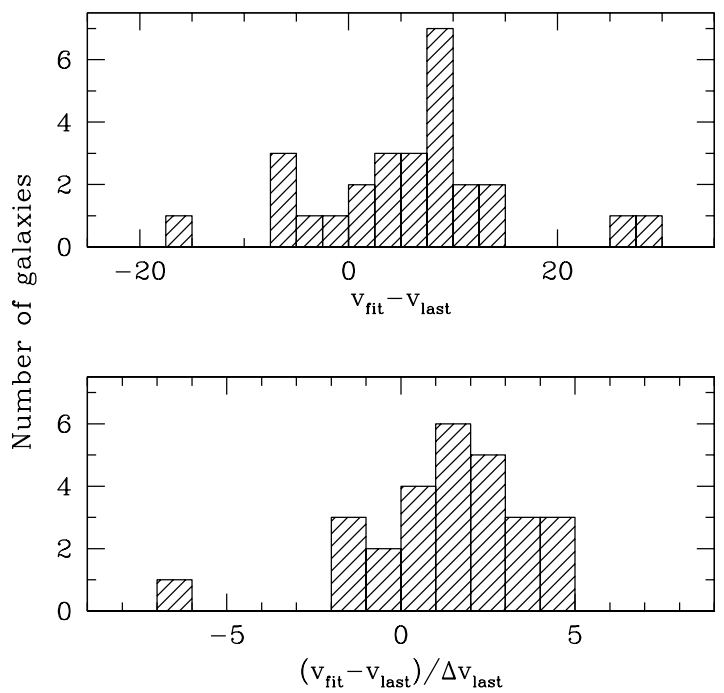

FIG. 2.- Histogram of the difference between the MOND-predicted rotation velocity and the observed velocity at the last measured point of the rotation curve (top panel). The bottom panel shows the same difference, but normalized by the uncertainty in the observed rotation velocity at the last measured point.

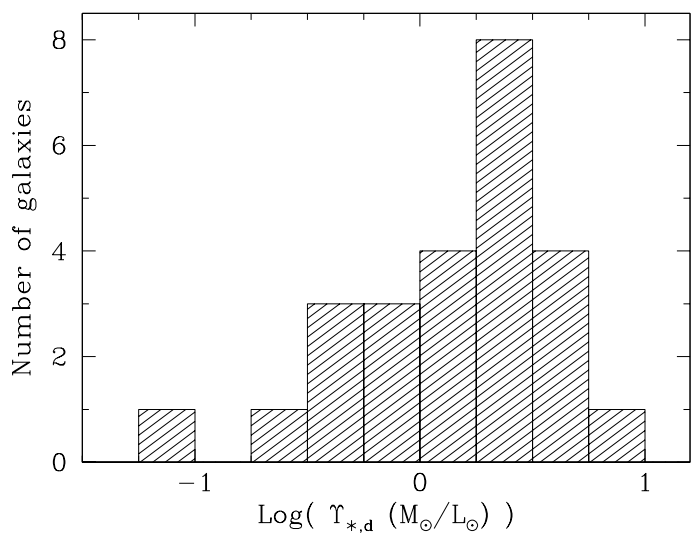

FIG. 3.- Histogram of best fit $\Upsilon_{*, d}$ values for models with only $\Upsilon_{*, d}$ as a free parameter. Two galaxies with best-fit $\Upsilon_{*, d}=0$ are not shown.

uncertainties, the thin solid line shows the contribution of the stellar disk, and the dashed line the contribution of the HI. The best fitting MOND rotation curve is indicated by the thick solid line.

A first inspection of the MOND fits presented in Figure 1 shows that roughly three quarters of the rotation curves are well fit by MOND, with only $\Upsilon_{*, d}$ as a free parameter. This is perhaps surprising, given the possible uncertainties due to inclination, distance, and asymmetries. A closer look shows that more than half of the galaxies deviate somewhat from the observed rotation rotation in a systematic way: the MOND curve predicts higher rotation velocities in in the outer regions, and lower in the central regions. This is seen clearly in e.g., UGC 6446 and UGC 12060, and it is also visible, although less pronounced, in e.g., UGC 731 and UGC 3371.

The degree to which MOND appears to predict higher velocities in the outer rotation curves is shown in Figure 2 in which a histogram of the difference between the observed and model velocities is presented, as well as one in which the differences have been normalized by the uncertainty in the rotation velocity of the last measured point. In 21 out of 27 
TABLE 2

BEST FIT PARAMETERS

\begin{tabular}{|c|c|c|c|c|c|c|c|c|c|c|}
\hline \multirow[b]{2}{*}{$\begin{array}{l}\text { UGC } \\
\text { (1) }\end{array}$} & \multirow{2}{*}{$\begin{array}{c}a_{0} \text { fixed } \\
\Upsilon_{R, \mathrm{x}} \\
(\mathrm{M} / \mathrm{L}) \odot \\
(2)\end{array}$} & \multicolumn{3}{|c|}{$a_{0}$ free } & \multicolumn{3}{|c|}{ distance free } & \multicolumn{3}{|c|}{ inclination free } \\
\hline & & $\begin{array}{c}a_{0} \\
\mathrm{~km}^{2} \mathrm{~s}^{-2} \\
\text { (3) }\end{array}$ & $\begin{array}{c}\Delta a_{0} \\
\mathrm{kpc}^{-1} \\
(4)\end{array}$ & $\begin{array}{c}\Upsilon_{R, \mathrm{f}} \\
(\mathrm{M} / \mathrm{L}) \odot \\
(5)\end{array}$ & $\begin{array}{l}d \\
(6)\end{array}$ & $\begin{array}{l}\Delta d \\
(7)\end{array}$ & $\begin{array}{c}\Upsilon_{R, \mathrm{~d}} \\
(\mathrm{M} / \mathrm{L}) \odot \\
(8)\end{array}$ & $\begin{array}{c}i \\
\circ \\
(9)\end{array}$ & $\begin{array}{c}\Delta i \\
\circ \\
(10)\end{array}$ & $\begin{array}{c}(\mathrm{M} / \mathrm{L}) \odot \\
(11)\end{array}$ \\
\hline UGC 731 & 4.3 & 1460 & 445 & 8.5 & 0.61 & 0.15 & 4.6 & 38 & 5 & 10.2 \\
\hline UGC 3371 & 2.8 & 2050 & 590 & 4.2 & 0.81 & 0.06 & 2.9 & 41 & 5 & 4.3 \\
\hline UGC 4173 & 0.1 & 550 & 350 & 0.6 & 0.43 & 0.08 & 0.1 & 24 & 5 & 0.7 \\
\hline UGC 4325 & 3.8 & 800 & 500 & 6.4 & 0.41 & 0.15 & 2.5 & 24 & 5 & 12.9 \\
\hline UGC 4499 & 0.3 & 3510 & 480 & 0.2 & 1.07 & 0.07 & 0.3 & 54 & 6 & 0.2 \\
\hline UGC 5005 & 0.5 & 1890 & 1100 & 1.3 & 0.78 & 0.21 & 0.8 & 35 & 6 & 1.3 \\
\hline UGC 5414 & 0.5 & 1880 & 1200 & 1.0 & 0.78 & 0.20 & 0.7 & 43 & 20 & 1.1 \\
\hline UGC 5721 & 3.9 & 9340 & 900 & 1.5 & 1.81 & 0.09 & 3.9 & 69 & 3 & 4.4 \\
\hline UGC 5750 & 0.3 & 5350 & 1600 & 0.0 & 1.32 & 0.42 & 0.0 & 63 & 30 & 0.3 \\
\hline UGC 6446 & 1.1 & 1710 & 300 & 2.1 & 0.69 & 0.09 & 1.3 & 39 & 3 & 2.5 \\
\hline UGC 7232 & 1.3 & 15300 & 7000 & 0.0 & 2.20 & 0.57 & 0.1 & 63 & 22 & 1.2 \\
\hline UGC 7323 & 0.8 & 4270 & 1740 & 0.5 & 1.16 & 0.25 & 0.8 & 57 & 18 & 0.5 \\
\hline UGC 7399 & 9.6 & 14200 & 1500 & 1.8 & 2.21 & 0.10 & 7.2 & 72 & 3 & 11.5 \\
\hline UGC 7524 & 0.9 & 1290 & 350 & 2.4 & 0.61 & 0.11 & 1.2 & 33 & 3 & 3.0 \\
\hline UGC 7559 & 0.0 & 650 & 680 & 3.2 & 0.48 & 0.27 & 0.8 & 32 & 9 & 3.4 \\
\hline UGC 7577 & 0.0 & 80 & 325 & 0.8 & 0.22 & 0.21 & 0.0 & 20 & 10 & 0.8 \\
\hline UGC 7603 & 0.8 & 6080 & 1100 & 0.2 & 1.40 & 0.13 & 0.4 & 63 & 10 & 0.3 \\
\hline UGC 8490 & 3.0 & 6690 & 700 & 1.3 & 1.51 & 0.08 & 2.6 & 67 & 3 & 2.6 \\
\hline UGC 9211 & 2.4 & 2110 & 1000 & 4.5 & 0.82 & 0.26 & 3.2 & 38 & 8 & 4.7 \\
\hline UGC 11707 & 1.2 & 1240 & 190 & 3.8 & 0.58 & 0.07 & 1.9 & 86 & 2 & 8.9 \\
\hline UGC 11861 & 2.0 & 2310 & 500 & 2.5 & 0.70 & 0.12 & 1.8 & 39 & 5 & 3.2 \\
\hline UGC 12060 & 1.8 & 690 & 270 & 5.6 & 0.32 & 0.10 & 1.6 & 21 & 4 & 13.7 \\
\hline UGC 12632 & 3.0 & 1190 & 390 & 8.0 & 0.54 & 0.14 & 3.6 & 30 & 5 & 11.4 \\
\hline F568-V1 & 5.4 & 1740 & 700 & 8.3 & 0.67 & 0.20 & 5.1 & 31 & 5 & 10.7 \\
\hline F574-1 & 3.0 & 950 & 460 & 6.5 & 0.34 & 0.14 & 2.1 & 90 & 2 & 47.5 \\
\hline F583-1 & 2.4 & 2590 & 990 & 2.9 & 0.91 & 0.20 & 2.5 & 52 & 18 & 2.9 \\
\hline F583-4 & 1.9 & 610 & 3500 & 6.9 & 0.24 & 0.68 & 1.6 & 24 & 11 & 17.2 \\
\hline
\end{tabular}

galaxies, MOND predicts higher than observed rotation velocities, whereas it predicts lower velocities in only 6 cases. The discrepancy is more than twice the uncertainty in the last measured point in 11 galaxies.

In Figure 3 we give the histogram of the best fit values for $\Upsilon_{*, d}$. The mass-to-light ratios span a large range, from 0 up to 10 . The average $R$-band $\Upsilon_{*, d}$ as derived from the best fits its $2 M_{\odot} / L \odot$.

\subsection{MOND fits with distance free}

Given that some of the adopted distances may be uncertain, it is possible that, for some of the galaxies for which MOND does not correctly predict the rotation curves, we have adopted an incorrect distance. We will investigate this by leaving the distance as a free parameter in the fits.

With distance as a free parameter, not only does the radial scale of the rotation curve change, but at the same time $v_{d} \propto 1 / \sqrt{d}$ and $v_{g} \propto \sqrt{d}$, where $d=\mathrm{D}_{\mathrm{MOND}} / \mathrm{D}_{a}$ is the fractional distance change. Strictly speaking, these relations are only correct if both the stellar and H I disks are infinitely thin. For disks with finite thicknesses, the shape of the rotation curves depends on the assumed thickness of the disk, and hence the shape of the rotation will change slightly if a difference distance is assumed.

The fits with distance as a free parameter are shown in Fig.1 as the dotted lines. From this figure it is clear that, with distance as a free parameter, good fits are obtained in virtually all cases. This demonstrates that a different distance can improve the fit. The best fit values for $d$, the fractional distance change, are listed in Table 2. The uncertainties on $d$ have been derived from the $68 \%$ confidence levels. However, because the errors on the rotation curves are non-Gaussian, because
$\mathrm{H}$ I the points are correlated, and because the rotation curves and the errors on the points may be affected by systematic effects, the confidence levels and the uncertainties derived from them should be considered estimates.

In Fig. 4 the distribution of $d$ is shown. The best fit fractional distance changes tend to be smaller than 1 . As can be seen in the bottom panel of Fig. 4, this is mostly due to galaxies at larger distances and for which the distances were determined from the Hubble flow. For these galaxies, the average fractional distance change $\langle d\rangle$ is 0.68 , with a dispersion of 0.29. Given that all these galaxies are at relatively large distances, it is unlikely that the Hubble flow distances are uncertain by that much, in particular because small $d$ are found even for galaxies at the largest distances.

For galaxies that are close by $(<10 \mathrm{Mpc}$; middle panel of Fig. 4), the best fit fractional distance changes appear to scatter around $d=1$ (i.e., no distance change). Such a large scatter is not unexpected because the galaxies are close-by and different methods have been employed to determine the distances to these galaxies.

\subsection{MOND fits with inclination free}

For most galaxies in our sample the inclination cannot be determined from the kinematics, because the rotation curves for most of the galaxies in this sample rise slowly without a well-defined flat part. As a result, for most galaxies in our sample the inclinations have been estimated from the morphology. For the galaxies in the SSAH sample, the inclinations mostly have been determined from the axis ratios of the outer isophotes (as described by Swaters \& Balcells 2002), except for those galaxies were the velocity field allowed a reliable estimate (see SSAH for details). For the LSB galaxies, 


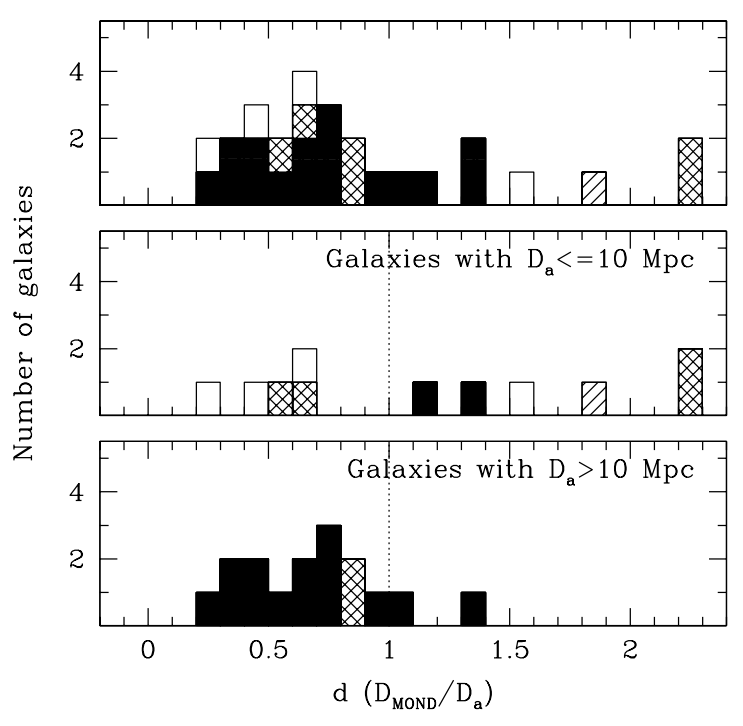

FIG. 4.- Histograms of $d$, the ratio between the best fit distance $\mathrm{D}_{\text {MOND }}$ found from a MOND fit with distance free, and $\mathrm{D}_{a}$, the adopted distance, for all galaxies (top panel), galaxies with $D_{a}<10 \mathrm{Mpc}$ (middle panel), and for galaxies with $D_{a}>10 \mathrm{Mpc}$ (bottom panel). The filled areas represent galaxies with distances determined from their $\mathrm{H}$ I recession velocities, the open areas represent galaxies with tip-of-the-RGB distances, the hatched areas represent brightest star distances, and the cross-hatched areas group membership distances.

the inclinations have been taken from de Blok \& McGaugh (1997), who used the H I maps and optical images to estimate the inclinations. Given how these inclinations have been derived, the inclinations of some of the galaxies in our sample are likely to be uncertain. To investigate whether some of MOND-predicted rotation curves can be improved by changing their inclinations, we have made MOND fits with inclination as a free parameter.

If the inclination is left free in the fits, the amplitude of the rotation curve changes with $\sin \left(i_{\mathrm{fit}}\right) / \sin i$, and at the same time, the contribution of the $\mathrm{H} \mathrm{I}$ and the stars to the rotation curve change with $\cos \left(i_{\text {fit }}\right) / \cos i$ (if optically thin). The latter is only valid for modest changes in the inclination, especially at high inclinations. When the changes are large, the rotation curves and density distributions may also change in shape.

In Table 2 we list the best fit inclinations. Uncertainties on the inclinations have been estimated from the $68 \%$ confidence levels in the fit, with the same caveats as described above for the fits with distance free. We do not show the best fits in Fig. 11 because the fits with inclinations free are almost indistinguishable from those with distance as a free parameter, except for UGCs $5721,7323,7399$, and 8490 , for which the fits are nearly indistinguishable from the original fit with only $\Upsilon_{*, d}$ as a free parameter.

In Figure 5 we plot the histogram of inclination changes, defined as $i-i_{\text {fit }}$. The inclination changes span a wide range, from $-30^{\circ}$ (more edge-on in MOND) to $+40^{\circ}$ (more faceon in MOND). For most galaxies the inclination changes are positive, which might be explained through bars. Note that in some cases the differences between the MOND and the adopted inclinations may be substantial, which means that for these fits the method used to fit the inclination may break down because the change in inclination is large enough to make the rotation curve shape change as well. However, we are mainly interested in investigating the general trends and not to get a measurement of the inclination change in this fit. If the best fit MOND inclination change for a particu-

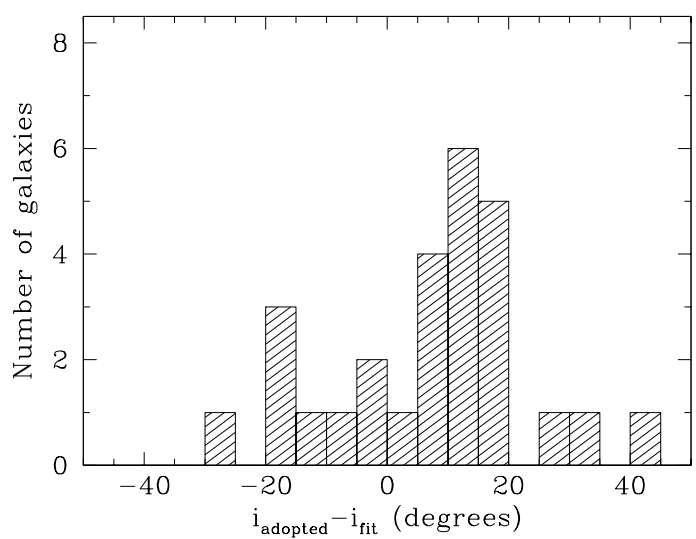

FIG. 5.- Histogram of inclination changes derived from fits to the rotation curve with inclination as a free parameter.

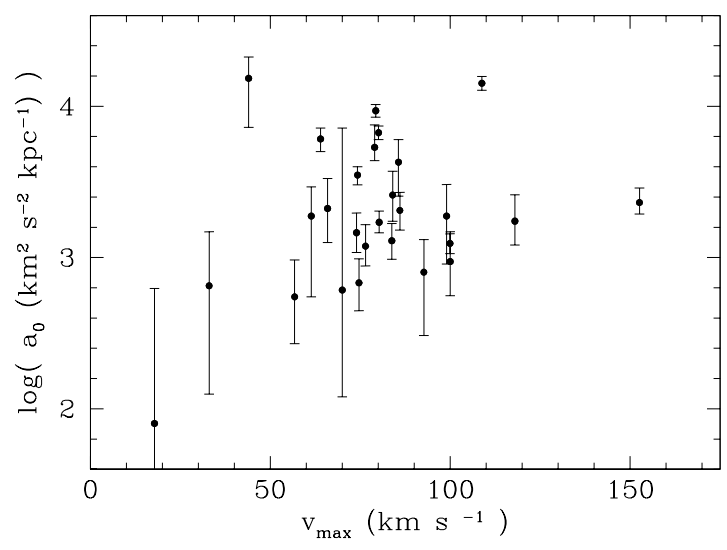

FIG. 6.- Plot of the best fit acceleration parameter $a_{0}$ versus the maximum rotation velocity $v_{\max }$. The correlation between these two parameters as reported by Lake (1989) is not found for the sample presented here.

lar galaxy is large, this likely indicates that MOND cannot be made compatible with that rotation curve by changing the galaxy's inclination, because for most galaxies the uncertainty in the inclinations are less than $10^{\circ}$.

\subsection{Fits with $a_{0}$ free}

Because $a_{0}$ is a universal parameter in MOND, the MOND fits should be made with $a_{0}$ fixed. However, leaving $a_{0}$ free provides both a means to measure $a_{0}$ (e.g., Begeman et al. 1991; Sanders \& McGaugh 2002), and a way to test MOND. If $a_{0}$ is truly universal, it should not depend on galaxy properties. To test this, we have made fits with $a_{0}$ as a free parameter. The best fit $a_{0}$ for each galaxy is listed in Table 2 along with an estimate of the uncertainties, for which the same caveat as mentioned above apply. The best fits are not shown separately in Fig. 11 because these fits are virtually indistinguishable from the fits with $d$ as a free parameter.

Lake (1989) reported that the acceleration parameter for his free fits correlated with the amplitude of the rotation curve. In Fig. 6 we have plotted our best fit $a_{0}$ versus the maximum rotation velocity $v_{\max }$. We find no evidence for a correlation between these parameters.

Interestingly, as can be seen in Fig. 7 there does appear to be a correlation between $a_{0}$ and surface brightness, in the sense that in galaxies with lower surface brightness lower values for $a_{0}$ are found. Taken at face value, the slope of the best fit to these points is -0.32 , i.e., $a_{0}$ drops by a factor of two for 

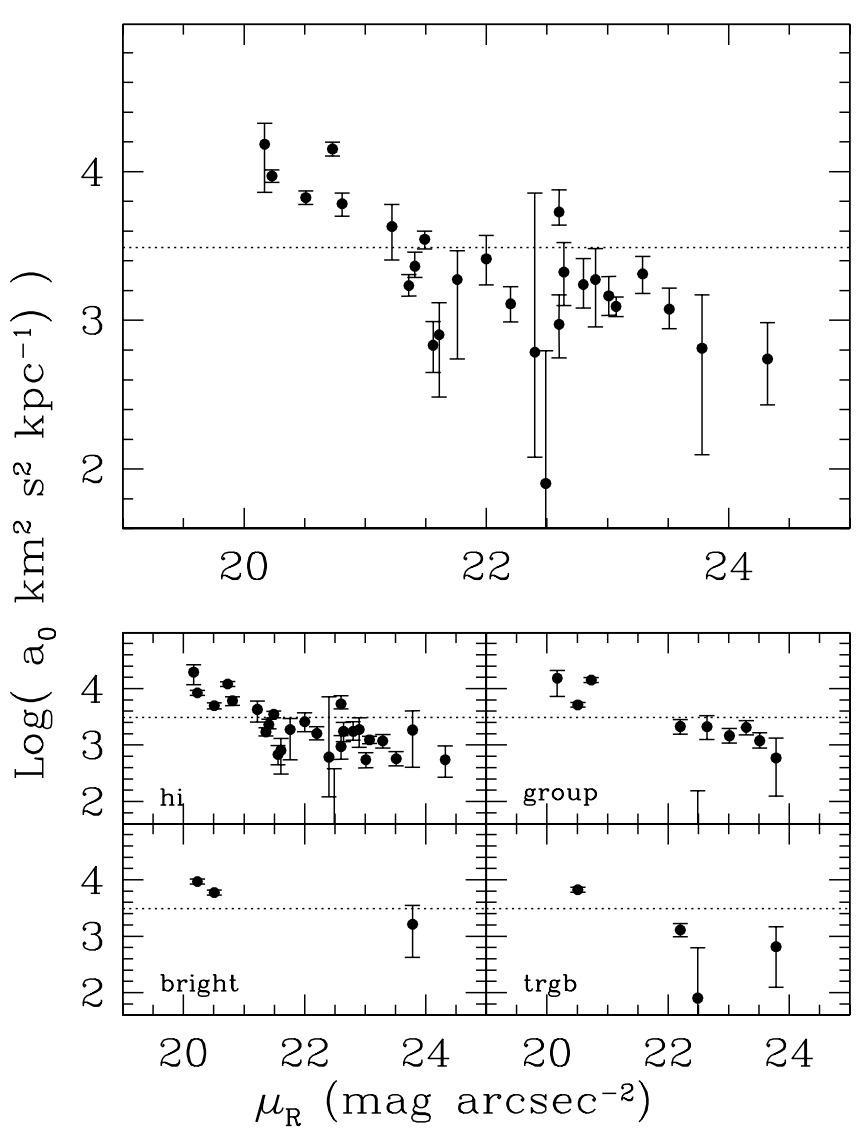

FIG. 7.- The top panel shows a plot of the best-fit acceleration parameter $a_{0}$ against the extrapolated central disk surface brightness $\mu_{R}$. There appears to be a correlation between these two parameters, although this apparent correlation depends strongly on a small number of points at the high surface brightness end (see Section 77. In the top panel, like in the rest of this paper, the adopted distances listed in Table 2 are used in the fits. However, for many galaxies more than one distance estimate is available (see e.g., Swaters \& Balcells 2002). In the bottom panel, the same correlation as in the top panel is shown for each four different methods of distance determination (Hubble flow, group membership, brightest star, and tip of the red giant branch). It is clear that the apparent trend does not depend on the method used to measure the distance.

each magnitude the surface brightness gets fainter. As can be seen in the bottom panels of Fig. 7 this trend does not depend on the method with which the distance was determined (for galaxies with different distance estimates we made multiple fits, but elsewhere only the fit results for the adopted distances as listed in Table 2 are presented).

The apparent trend between the acceleration parameter and the central disk surface brightness depends strongly on the galaxies brighter than $\mu_{R}=21 \mathrm{mag} \operatorname{arcsec}^{-2}$. We cannot rule out that this trend is the result of uncertainties (see sections 6 and 7 .

\section{REMARKS ON INDIVIDUAL GALAXIES}

Below we discuss the fits shown in Fig. 1. Unless otherwise indicated, the 'MOND curve' refers to the fit with only $\Upsilon_{*, d}$ as a free parameter.

UGC 731.- Swaters et al. (1999) showed that this galaxy is lopsided in its kinematics, with a rotation curve that rises more steeply on one side of the galaxy than on the other. The average rotation curve, as presented in Figure 1 is in good agreement with MOND, except that in the outer parts MOND predicts somewhat higher rotation velocities.
UGC 3371. - The rotation curve of this galaxy has been derived from a high-resolution $\mathrm{H} \alpha$ velocity field (see Swaters et al. 2003b). The MOND curve is in good agreement with the observed rotation curve, although it is slightly above the outer few points.

UGC 4173. - The MOND curve based on the H I alone already predicts significantly higher rotation velocities than are observed. This galaxy has an optical bar, with a faint surrounding disk, making the inclination difficult to determine. An inclination of $25^{\circ}$ to $30^{\circ}$ is consistent with the $\mathrm{H}$ I morphology, and would make this galaxy consistent with MOND, and also bring it closer to the Tully-Fisher relation.

UGC 4325. - The MOND curve and the data agree fairly well. The MOND curve falls slightly above the observed points in the outer parts.

UGC 4499. - There is good general agreement between the MOND prediction and the observed rotation curve, although there is considerable scatter in derived rotation velocities near the center.

UGC 5005. - There is good agreement between the data and the MOND curve.

UGC 5414. - The MOND curve is in excellent agreement with the rotation curve.

UGC 5721.- For this compact, high-surface brightness galaxy, the MOND curve fails to describe the observed rotation curve. Even with the inclination or the distance as a free parameter, no good fit can be found. There is evidence of twisting isophotes in the central regions (see Swaters \& Balcells 2002), perhaps indicating the presence of a bar. If so, the associated noncircular motions could have affected the derived rotation curve.

UGC 5750. - The MOND curve is in good general agreement with the observed rotation curve.

UGC 6446 - The MOND curve falls below the observed rotation curve in the central parts, and above the rotation curve in the outer parts. If this galaxy is part of the Ursa Major cluster (e.g., Tully et al. 1996), the increased distance of $18.6 \mathrm{Mpc}$ (Tully \& Pierce 2000) would make this galaxy less compatible with MOND. However, this galaxy is on the edge of the boundary taken to define the UMa cluster in both velocity and angle on the sky, so it may not be a genuine cluster member. In this paper, we have adopted a distance of $12.8 \mathrm{Mpc}$.

UGC 7232. - The MOND curve and the data agree fairly well.

$U G C$ 7323. - The MOND curve is somewhat higher than the observed curve in the central parts, but there is good general agreement.

UGC 7399.- For this compact galaxy, the MOND curve does not agree with the observed rotation curve, as it predicts a much steeper central gradient than is observed in the rotation curve. The bar in this galaxy may have affected the derived rotation curve.

$U G C$ 7524. - The MOND curve and the observed rotation curve for this well-resolved galaxy agree well, except in the outer points.

UGC 7559. - The MOND curve agrees well with the observed curve in the inner parts, but it predicts significantly higher-than-observed rotation velocities in the outer parts. Like UGC 5721, this galaxy appears to have a bar, and the associated noncircular motions could have affected the derived rotation curve.

UGC 7577. - The observed rotation curve of this galaxy can be explained by gas and stars alone, even with Newtonian gravity. Consequently, the MOND curve predicts sig- 
nificantly higher rotation velocities in the outer parts. A lower distance and a lower inclination would help to make the MOND prediction more compatible with the observed curve.

$U G C$ 7603. - Although the MOND curve falls somewhat below the observed rotation curve in the outer parts, there is good general agreement.

UGC 8490. - The MOND prediction for this galaxy falls systematically below the observed flat part of this rotation curve. A larger distance makes this rotation curve more consistent with the MOND curve, although the MOND curve still falls short of the observed rotation curve near the turnover in the rotation curve. Because of the large warp in this galaxy, the shape and amplitude of the rotation curve are uncertain.

UGC 9211. - The curve predicted by MOND and the observed curve are in good agreement.

UGC 11707. - The rotation curve predicted by MOND falls below the inner rotation curve, and above the outer rotation curve.

UGC 11861.- The MOND curve and the observations are in good agreement.

UGC 12060.- The rotation curve as predicted by MOND falls below the observed one in the inner parts of the rotation curve, and is above the observed one in the outer parts. An inclination of $20^{\circ}$ in stead of $40^{\circ}$ is required for agreement between MOND and the observations. Such a low inclination is consistent with the optical morphology of this galaxy, but appears inconsistent with the Tully-Fisher relation.

UGC 12632. - The rotation curve predicted by MOND falls below the observed curve in the inner parts and above the observed curve in the outer parts.

F568-V1.- There is good agreement between the MOND prediction and the observed rotation curve.

F574-1. - Except for the one outermost point in the MOND prediction, which may well be due to uncertainties in the contribution of the $\mathrm{H}$ I to rotation curve, the MOND curve and the observed one are in good agreement.

F583-1. - Although the MOND curve falls slightly above the observed velocities in the center, and slightly below at intermediate radii, there is good general agreement between the observed rotation curve and the MOND prediction.

F583-4. - There is good agreement between the observed and the MOND predicted rotation curves.

\section{UNCERTAINTIES}

As is clear from the notes on individual galaxies and the fits shown in Fig. 1 for about a quarter of the galaxies in the sample there are noticeable discrepancies between the observed and the predicted curves, and in a few instances there are significant inconsistencies. On the one hand, these inconsistencies may signal a problem for MOND, but, on the other, they might be the result of uncertainties associated with the galaxies in this sample. Because MOND fits are essentially oneparameter fits, with only the mass-to-light ratio of the stars as a free parameter, it is much more sensitive to observational uncertainties than fits with dark matter halos. For such fits with dark matter halos, any uncertainties in the rotation curve or in the contribution of the stars and gas, for example as a result of uncertainties in distance or inclination, can be accommodated by changing the parameters of the dark matter halo. The MOND fits, which are directly tied to the distribution of the gas and stars, cannot accommodate these uncertainties. As a result, uncertainties in e.g., the rotation curves, the distances, or the inclinations can lead to apparently good dark matter fits (although likely with incorrect halo parameters), whereas the same uncertainties can lead to very poor MOND predictions of the rotation curves. It is therefore imperative to consider the uncertainties that may play a role for the data presented here before we can discuss the implications of our results for MOND.

\subsection{Observational uncertainties}

Given that the sample presented here consist in large part of nearby dwarf galaxies, uncertainties in distances will likely play a role. For those nearby galaxies, the distances have been determined with different methods: Hubble flow, group membership, brightest stars, and tip of the red giant branch. Some of these methods can produce uncertain distance measurements. As we have shown in this paper, the MOND fits can be improved by adopting different distances. It is likely that in individual cases incorrect distance estimates have contributed to poor MOND predictions of the rotation curves. However, it is unlikely that changes in distance alone can explain all the poor predictions, because that would require the galaxies in our sample to be preferentially closer, even galaxies at large distances which presumably have more accurate distances.

Another important factor that may contribute to uncertainties in the MOND fits are the inclinations of the galaxies in our sample. As described in Section 4.3. many of the galaxies in our sample have irregular appearances and slowly rising rotation curves, making it difficult to determine their inclinations from either their morphology or their kinematics. Moreover, towards more face-on galaxies, intrinsic noncircular shapes may lead to an overestimate of the inclination, and towards higher inclinations the uncertainties in the intrinsic thicknesses of these galaxies may affect the derived inclinations. Thus, the inclinations of the galaxies in our sample may be uncertain.

We have shown that the MOND fits can significantly be improved by adopting different inclinations. Moreover, for some galaxies, like UGC 4173 and UGC 12060, the MONDpreferred inclinations not only improve the MOND fits, but also are compatible with the galaxies morphology and kinematics. In the case of UGC 4173, the MOND-preferred inclination also brings the galaxy closer to the Tully-Fisher relation. However, for most galaxies the required changes in inclination are larger than the expected uncertainties. In addition, if changes in inclination would be the main cause of the poor MOND predictions of the rotation curves, then the majority of the galaxies in our sample would have to be more face-on than the inclinations reported in Table 1 . This could be possible if the galaxies in our sample have significant intrinsic ellipticities or strong bars. However, to explain differences of up to $20^{\circ}$ even at inclinations of about $50^{\circ}$, we estimate an intrinsic ellipticity of 0.35 is needed. Such large intrinsic ellipticities are not seen among late-type disk galaxies (e.g., Schoenmakers et al. 1997; Andersen et al. 2001), although they might occur in low luminosity systems (Sung et al. 1998). However, there is little evidence of the perturbations in the velocity fields associated with such strong ellipticities (Schoenmakers et al. 1997) in the velocity fields presented in Swaters et al. (2002). It is therefore unlikely that all the poor MOND predictions can be explained by uncertainties in the inclination alone, but it is likely that in some individual cases incorrect adopted inclinations can be the main reason for poor MOND predictions.

Naturally, it is also possible that both the adopted inclination and distance are incorrect. We investigated this in our fitting process but found that in all but a few of our fits, distance 
and inclination are generally strongly covariant, and in most cases it was not possible to constrain both of these parameters simultaneously. Still, when compared to leaving only the distance or the inclination free in the fits, if both are left free, on average smaller changes are required to make the MOND-predicted curve more compatible with observed rotation curve. At the same time, the required changes are still systematic, i.e., on average the galaxies still need to be closer and more face-on.

In addition to the uncertainties in distance and inclination, other factors may also play a role. These include beam smearing, noncircular motions, morphological asymmetries, corrections for asymmetric drift, and uncertainties in the photometric calibration. Even though the $\mathrm{H}$ I rotation curves presented here have been corrected for beam smearing (see SSAH), the inner rotation curve shape may be uncertain simply due to lack of resolution. For many galaxies in our sample highresolution $\mathrm{H} \alpha$ rotation curves are available to mitigate this effect, but $\mathrm{H} \alpha$ rotation curves themselves may be uncertain because they are determined along a one-dimensional slice and not from the velocity field as a whole and hence are more susceptible to noncircular motions. In addition, the rotation curves presented here have not been corrected for asymmetric drift because it was deemed not to be important. Another potential factor are morphological and kinematical asymmetries. Not only can these affect the derived baryon distributions and rotation curves, they are also incompatible with the underlying assumption of axisymmetry used in calculating the MOND-predicted rotation curves. All these factors combined could result in a derived rotation curve that is not representative of the true circular velocity.

\subsection{Fitting uncertainties}

In addition to observational uncertainties, the quality of the fits may be affected by the fitting process and the associated assumptions. An example is the adopted thickness for the H I and stellar disks. Here, for ease of comparison with other studies, we have assumed that the gaseous and stellar disks are infinitely thin. Compared to a stellar disk that is assumed to have a scale height of 0.2 disk scale lengths, as was adopted by Swaters (1999), the rotation curve calculated for an infinitely thin disk rises more steeply, on average by $15 \%$, but reaching up to $40 \%$ for galaxies with central concentrations of light. The amplitude in the thin disk case is about 5-10\% higher than in the thick disk case. Thus, taking a thick disk in stead of a thin disk would results in M/Ls that are $10 \%$ to $20 \%$ higher, and values for $a_{0}$ that are correspondingly lower. The rotation curve of the $\mathrm{H} \mathrm{I}$ is less sensitive to the adopted thickness. The difference between an infinitely thin disk and a disk with a scale height of 0.2 optical disk scale lengths is in general well below 5\%, and hence the choice of thickness of the $\mathrm{H}$ I disk has little influence on the fits.

Another uncertainty on the results is the adopted form of $\mu$ (see Eq. 1), that describes the transition between the Newtonian and MOND regimes. In the results reported in the Tables and Figures in this paper, we have used the commonly used form given in Eq. 2] We have also tried the form:

$$
\mu(x)=x /(1+x),
$$

as used by Famaey \& Binney (2005) and McGaugh (2008). This revised transition formula results in more noticeable deviations from Newtonian gravity at higher accelerations. This has an effect on the fits, and we looked in particular in terms of the possible correlation between $a_{0}$ and surface brightness as shown in Fig. [7] When Eq. 5] is used, galaxies that are deeper in the MOND regime (i.e., the LSB galaxies) tend to end up with lower values for $a_{0}$, whereas the derived values for $a_{0}$ change little for high surface brightness galaxies.

The fact that some of the rotation curves in our sample have been derived from a combination of $\mathrm{H} \mathrm{I}$ and $\mathrm{H} \alpha$ data might have affected our fit results, because the long-slit $\mathrm{H} \alpha$ data sample only a single slice through the velocity field, making them more sensitive to the effects of noncircular motions. In addition, the higher spatial sampling in the galaxy centers might bias the fits. However, any such bias would, at least partly, be offset by the usually larger uncertainties on the $\mathrm{H} \alpha$ rotation velocities. Even though such a bias could have played a role, we find it does not for the galaxies in our sample. For all galaxies for which we have hybrid $\mathrm{H} \mathrm{I}-\mathrm{H} \alpha$ rotation curves, we have made fits to the $\mathrm{H}$ I-only rotation curves and found no significant differences. This is also illustrated by the fact that there are no systematic differences between the galaxies in our sample for which we have $\mathrm{H}$ I-only rotation curves, and those for which we have hybrid rotation curves.

Perhaps the biggest uncertainties on the derived parameters are from the fitting process itself. The values reported in Table 2 are best-fit parameters. However, in some cases, the best-fit values suggest large changes in distance, inclination, or $a_{0}$, even though the nominal values provide fits that are nearly as good in a $\chi^{2}$ sense (e.g, UGC 5414 and F583-4). In most of these cases, the estimated uncertainties on the derived parameters are large, thereby indicating that a large range in parameter space produces fits of similar quality. In a number of cases, however, the best-fit values for the derived parameters are off from the nominal value by a large amount, even when a visual comparison of the MOND fits and the fits with additional free parameters suggests that both fits are comparable in quality. Examples of this are UGC 3371, UGC 11861, and F574-1. In light of the uncertainties on the rotation velocity uncertainties, as was also mentioned in Sec. 4 the best-fit values derived from the fits should not be taken at face value.

\section{DISCUSSION}

We have found that the rotation curves as predicted by MOND for the sample of dwarf and LSB galaxies presented in this paper are generally in good agreement with the observed rotation curves for roughly three quarters of the sample. This is remarkable, given that MOND is a one parameter fit with only $\mathrm{M} / \mathrm{L}$ as a free parameter. It is even more remarkable, in light of the uncertainties associated with a sample of low surface brightness and dwarf galaxies, as was discussed in more detail in the previous section. Given these uncertainties, a fraction of around a quarter of galaxies for which MOND does not adequately predict the observed rotation curves does not seem unexpected, and hence the differences between the observed rotation curves and the MOND fits may not signal a failure of MOND, but rather reflect the uncertainties associated with the galaxies in this sample.

Despite these uncertainties, there are a number of interesting results from our study. One is that the MOND fits appear to deviate in a systematic way from the observed rotation curves. In almost $80 \%$ of the galaxies (21 out of 27) the MOND curve predicts higher rotation velocities in the outer parts than are observed. In $40 \%$ of the galaxies in our sample, MOND predicts rotation velocities that are higher by more than twice the uncertainty in the rotation velocity of the last measured point of the rotation curve. As we have shown, 
these discrepancies can be explained if these galaxies have different inclinations or distances. To explain the apparent systematic deviations between the MOND curves and the observed rotation curves in terms of changes in distance or inclination, the galaxies in our sample need to be preferentially closer, need to have preferentially lower inclinations, or both, which seems unlikely.

An alternative explanation for the fact that most MONDpredicted curves tend to have higher rotation velocities in the outer parts than is observed, is that the value for $a_{0}$ is preferentially lower. Of course, in MOND $a_{0}$ is a universal constant and should not vary from galaxy to galaxy. In the MOND fits presented here, we have adopted $a_{0}=3080 \mathrm{~km}^{2} \mathrm{~s}^{-2} \mathrm{kpc}^{-1}$ (e.g., Sanders \& McGaugh 2002). If we average the $a_{0}$ derived from our fits with the acceleration parameter free, we find $a_{0}=3350 \mathrm{~km}^{2} \mathrm{~s}^{-2} \mathrm{kpc}^{-1}$, consistent with the value used by Sanders \& McGaugh (2002). However, as can be seen in 7 most of our fits result in low values for $a_{0}$. Excluding the three highest values for $a_{0}$, we find $a_{0}=2150 \mathrm{~km}^{2} \mathrm{~s}^{-2} \mathrm{kpc}^{-1}$. Thus, a somewhat lower value for $a_{0}$ would, on average, lead to improved fits for the galaxies in our sample.

We also have found what appears to be a correlation between $a_{0}$ and surface brightness. Such a trend, if real, would be a problem for MOND, because $a_{0}$ is a universal constant. However, it seems that this correlation is largely caused by a small number of galaxies at the high surface brightness end, for which large values for $a_{0}$ were found: UGC 5721, UGC 7232, UGC 7399, UGC 7603, and UGC 8490. As we noted in Sec. 5. UGC 5721 and UGC 7399 are compact galaxies with a central bar, and hence the inner rotation curve could be affected by noncircular motions. UGC 8490 has a strong warp, making the outer rotation curve less certain. UGC 7232 only has four points in its rotation curve and large uncertainties on the fitted value of $a_{0}$. Considering these factors, it is possible that the apparent correlation is the result of uncertainties, and not of a true underlying relation.

Previous studies of MOND rotation curves that included LSB galaxies in their samples have not reported a trend between surface brightness and $a_{0}$. De Blok \& McGaugh (1998) analyzed the $\mathrm{H}$ I rotation curves presented in de Blok et al. (1996) in the context of MOND, and found most galaxies to be consistent with MOND, although for a few adjustments to distance and inclination had to be made. The sample presented in Sanders \& Verheijen (1998) contained 12 LSB galaxies, but the authors did not report a trend between $a_{0}$ and surface brightness. Neither does an inspection of the LSB galaxies in their sample show the prediction by MOND of systematically faster rotation in the outer rotation curves, like is seen in our sample, except for UGC 6446, which is a galaxy also included in this sample. However, our sample extends more than a magnitude deeper in surface brightness, making the sample presented here more sensitive to effects that may play a role at low surface brightness.

Interestingly, it may be possible to explain, within the context of MOND, why at least some galaxies would appear to have a low value for $a_{0}$ when $a_{0}$ is left as a free parameter in rotation curve fits. In the results presented here, we have only considered the accelerations within the galaxies themselves. However, whether an object is in the MOND regime is determined by the overall acceleration, which includes external effects (Milgrom 1983a,b). Thus, if an object, with internal accelerations that would place it in the MOND regime, is placed in a Newtonian external acceleration that is larger than $a_{0}$, its kinematics would be entirely Newtonian. If one were to fit $a_{0}$ in that case, one would derive a value of zero, because the object would never enter the MOND regime. Of course, the effects of the external fields are much smaller for the galaxies in our sample. Even for the galaxy in our sample with the closest neighbor (UGC 7577, near NGC 4449), the external acceleration is estimated to be $1 \times 10^{-9} \mathrm{~cm} \mathrm{~s}^{-2}$ (assuming a projected distance of $14.4 \mathrm{Mpc}$ ). Even though the amplitude of the external field is difficult to determine because it depends on the details of the galaxy environment, it can only lower the value of $a_{0}$ in fits where $a_{0}$ is left free. Moreover, any external fields will have the strongest effects in LSB galaxies, where the internal accelerations are lowest. Thus, while external fields may contribute to the apparent correlation between surface brightness and $a_{0}$, it is difficult to establish this quantitatively.

Given the uncertainties in the distances, the inclinations, other observational uncertainties, uncertainties in the fitting process, and possible effects of external fields, it seems that the correlation between surface brightness and $a_{0}$ may not be significant. Perhaps more certain is the finding that the galaxies in the sample presented here suggest a lower value for $a_{0}$ than the adopted value of $3080 \mathrm{~km}^{2} \mathrm{~s}^{-2} \mathrm{kpc}^{-1}$.

To thoroughly test MOND in the LSB and dwarf galaxy regime, galaxies are needed with reliable distance measurements and inclinations, well-resolved rotation curves and symmetric appearance, both in morphology and kinematics. None of the galaxies in our sample meet all these criteria simultaneously. However, there are four galaxies that meet all but one: UGC 3371, UGC 6446, UGC 7524, and UGC 12632. UGC 7524 is the only one with a reliable distance measurement from the TRGB (Karachentsev et al. 2003), but it has asymmetric kinematics (Swaters et al. 1999).

\section{SUMMARY AND CONCLUSIONS}

We have presented MOND fits for a sample of 27 dwarf and LSB galaxies. MOND is remarkably successful at predicting the general shape of the rotation curves in this sample. Only for approximately a quarter of the galaxies MOND does not adequately predict the observed rotation curves. This is remarkable given the uncertainties associated with the galaxies in this sample, especially the uncertainties in distance and inclination. Close inspection of the fits indicates that for almost $80 \%$ of the galaxies MOND predicts higher velocities in the outer rotation curves than is observed. Although this could be the result of uncertainties, this systematic deviation can also be explained if the MOND acceleration parameter is slightly lower than usually assumed, with a value of $a_{0}=2150 \mathrm{~km}^{2}$ $\mathrm{s}^{-2} \mathrm{kpc}^{-1}$ (or $0.7 \times 10^{-8} \mathrm{~cm} \mathrm{~s}^{-2}$ ). We find that there appears to be some evidence of a correlation between $a_{0}$ and the central extrapolated disk surface brightness $\mu_{R}$, but this possible correlation depends heavily on a few galaxies at high surface brightness, whose rotation curves may be uncertain because of bars or warps. At the low surface brightness end, the derived values of $a_{0}$ could be be biased towards low values if external fields are important. Overall, the uncertainties associated with this sample make it difficult to draw strong conclusions. Improved distances and inclination estimates for these galaxies could make it possible to test MOND more strongly. 


\section{REFERENCES}

Andersen, D. R., Bershady, M. A., Sparke, L. S., Gallagher, J. S., III, \& Wilcots, E. M. 2001, ApJ, 551, L131

Begeman, K. G., Broeils, A. H., \& Sanders, R. H. 1991, MNRAS, 249, 523

Bekenstein, J., \& Milgrom, M. 1984, ApJ, 286, 7

Blais-Ouellette, S., Amram, P., \& Carignan, C. 2001, AJ, 121, 1952

Bottema, R., Pestaña, J. L. G., Rothberg, B., \& Sanders, R. H. 2002, A\&A, 393, 453

Clowe, D., Bradač, M., Gonzalez, A. H., Markevitch, M., Randall, S. W., Jones, C., \& Zaritsky, D. 2006, ApJ, 648, L109

de Blok, W. J. G., \& Bosma, A. 2002, A\&A, 385, 816 (dBB)

de Blok, W. J. G., \& McGaugh, S. S. 1997, MNRAS, 290, 533

de Blok, W. J. G., \& McGaugh, S. S. 1998, ApJ, 508, 132

de Blok, W. J. G., van der Hulst, J. M., \& Bothun, G. D. 1995, MNRAS, 274, 235

de Blok, W. J. G., McGaugh, S. S., \& van der Hulst, J. M. 1996, MNRAS, 283,18

de Blok, W. J. G., McGaugh, S. S., \& Rubin, V. C. 2001, AJ, 122, 2396 (dBMR)

Famaey, B., \& Binney, J. 2005, MNRAS, 363, 603

Karachentsev, I. D., et al. 2003, A\&A, 398, 467

Lake, G. 1989, ApJ, 345, L17

Lucy, L. B. 1974, AJ, 79, 745

McGaugh, S. S. 2008, ApJ, 683, 137

McGaugh, S. S., \& Bothun, G. D. 1994, AJ, 107, 530

Méndez, B., Davis, M., Moustakas, J., Newman, J., Madore, B. F., \& Freedman, W. L. 2002, AJ, 124, 213

Milgrom, M. 1983a, ApJ, 270, 365

Milgrom, M. 1983b, ApJ, 270, 371

Milgrom, M. 1986, ApJ, 302, 617

Milgrom, M. 1991, ApJ, 367, 490
Milgrom, M., \& Braun, E. 1988, ApJ, 334, 130

Milgrom, M., \& Sanders, R. H. 2007, ApJ, 658, L17

Milgrom, M., \& Sanders, R. H. 2008, ApJ, 678, 131

Sakai, S., et al. 2000, ApJ, 529, 698

Sanders, R. H. 1996, ApJ, 473, 117

Sanders, R. H. 1999, ApJ, 512, L23

Sanders, R. H., \& McGaugh, S. S. 2002, ARA\&A, 40, 263

Sanders, R. H., \& Noordermeer, E. 2007, MNRAS, 379, 702

Sanders, R. H., \& Verheijen, M. A. W. 1998, ApJ, 503, 97

Schoenmakers, R. H. M., Franx, M., \& de Zeeuw, P. T. 1997, MNRAS, 292, 349

Sung, E.-C., Han, C., Ryden, B. S., Patterson, R. J., Chun, M.-S., Kim, H.-I., Lee, W.-B., \& Kim, D.-J. 1998, ApJ, 505, 199

Swaters, R. A. 1999, PhD thesis, Rijksuniversiteit Groningen

Swaters, R. A., \& Balcells, M. 2002, A\&A, 390, 863

Swaters, R. A., Schoenmakers, R. H. M., Sancisi, R., \& van Albada, T. S. 1999, MNRAS, 304, 330

Swaters, R. A., Madore, B. F., \& Trewhella, M. 2000, ApJ, 531, L107

Swaters, R. A., Madore, B. F., van den Bosch, F. C., \& Balcells, M. 2003a, ApJ, 583, 732 (SMBB)

Swaters, R. A., Verheijen, M. A. W., Bershady, M. A., \& Andersen, D. R. 2003b, ApJ, 587, L19 (SVBA)

Swaters, R. A., Sancisi, R., van Albada, T. S., \& van der Hulst, J. M. 2009 , A\&A, 493, 871 (SSAH)

Tully, R. B., \& Pierce, M. J. 2000, ApJ, 533, 744

Tully, R. B., Verheijen, M. A. W., Pierce, M. J., Huang, J.-S., \& Wainscoat, R. J. 1996, AJ, 112, 2471

van der Hulst, J. M., Skillman, E. D., Smith, T. R., Bothun, G. D.,

McGaugh, S. S., \& de Blok, W. J. G. 1993, AJ, 106, 548

Warmels, R. H. 1988, A\&AS, 72, 427 